\title{
Experimental and numerical assessment of the characteristics describing superelasticity in shape memory alloys - influence of boundary conditions
}

\author{
Jakub Bryła ${ }^{1}$ and Adam Martowicz ${ }^{1^{*}}$ \\ ${ }^{1}$ AGH University of Science and Technology, Faculty of Mechanical Engineering and Robotics, Department of Robotics and \\ Mechatronics, Mickiewicza 30, 30-059 Krakow, Poland
}

\begin{abstract}
Relatively recent discovery of shape memory alloys (SMA) justifies ongoing research on their properties and an attempt to explain the physical phenomenon responsible for the characteristic behaviour of SMA. Moreover, there have been reported many successful commercial SMA applications to medical cases, mostly based on superelasticity. Even though a wide application range is confirmed, its further contribution growth is currently not seen - mostly due to deficiency of reliable modelling techniques. Recently, lively discussion in the SMA academic community is observed, which deals with modelling issues and numerical implementation.

Considering the current trends, the authors of the work make an attempt at qualitative analysis of the material properties for superelasticity. The material characteristics - found using static stretching tests - are sensitive to the variation of local stresses induced in the area where a SMA sample is mounted in a fatigue testing machine. As shown, the phenomena present at the clamping area seem to initiate and govern the process of the solid phase transformation within the entire SMA body. The overall objective of the presented research is to assess the influence of the above stated boundary conditions on the properties of selected types of SMA, using both experimental and numerical results.
\end{abstract}

\section{Introduction}

Shape memory alloys (SMA) belong to smart materials. Their unique mechanical properties reveal through the two-way martensitic phase transition activated by force and thermal excitations [1-3]. This nanoscale phenomenon introduces the specific macroscale behaviour of SMA, namely one- and two-way shape memory effects, and superelasticity. In general, the mentioned effects cause the nonlinear mechanical response of SMA. During each of them the transformation between solid phases occurs, where martensitic and austenitic structures have the main role. The differences in material properties of both phases cause unique behaviour of SMA. Nature of structure transformations is briefly described below for better understanding of the physical background of the SMA behaviour. Next, the superelasticity is considered due to its adoption in many practical applications. Further details regarding the one- and two-way shape memory effects may be found in the authors' former works [4-7].

As previously mentioned, the martensite and austenite phases have different material properties. The most important, from practical reasons, are their values of the Young's modulus. Martensite is much less stiff than austenite. Moreover, the existence conditions of both phases are clearly identified. Therefore, the martensite phase appears in SMA at low temperature, contrary to the conditions when austenite may be found. Note that, the temperature below/above which only one solid phase in material is observed (martensite/austenite) varies according to the direction of temperature changes. Hence, a hysteresis in the material response is observed. The relation between phases percentage contribution rates and ambient temperature is schematically shown in Fig. 1.

Therefore, the four temperatures describe the conditions for the phase transformations between martensite and austenite in SMA: $\mathrm{M}_{\mathrm{s}}, \mathrm{M}_{\mathrm{f}}, \mathrm{A}_{\mathrm{s}}$ and $\mathrm{A}_{\mathrm{f}}$, respectively marked in Fig. 1 . As a matter of fact, these temperatures are functions of stress. It means that the above explanation refers to the case when mechanical constraints are not applied to an element made of SMA. The values of the mentioned characteristic temperatures rise along with greater stress generation in the material. This phenomenon has a significant impact on the SMA behaviour at specified temperature range, for which only austenite exists in a non-loaded element: $M_{d}>T>A_{f}$. Consequently, the values of the characteristic temperatures rise after applied mechanical constraints to a SMA element. Generation of a fairly high stress field causes the situation when $\mathrm{T}<\mathrm{M}_{\mathrm{s}}$, which finally leads to initiation of phase transformation process. The phenomenon is called as superelasticity.

*Corresponding author: adam.martowicz@agh.edu.pl 


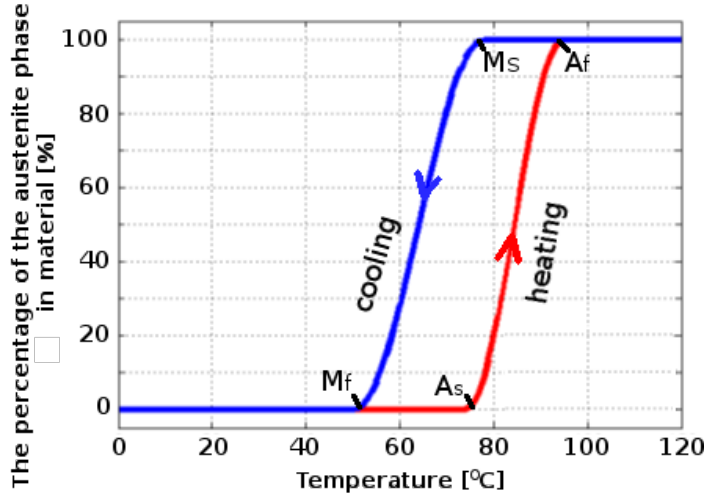

Fig. 1. Relation between phases contribution rates and temperature changes.

The superelasticity is a reversible effect. It means that martensite transforms back to austenite when a SMA component is unloaded. The full load cycle is shown in Fig. 2. One can observe that the stress-strain path depends on the constraint direction [8]. As a result the hysteresis occurs. This fact is widely applied when designing damping systems [9]. Application possibilities for a bumper made of SMA were also verified by the authors of the present work by performing uncertainty analysis [4-5]. Another application area is medicine, showing how crucial the existing features for popularization the SMA devices are.

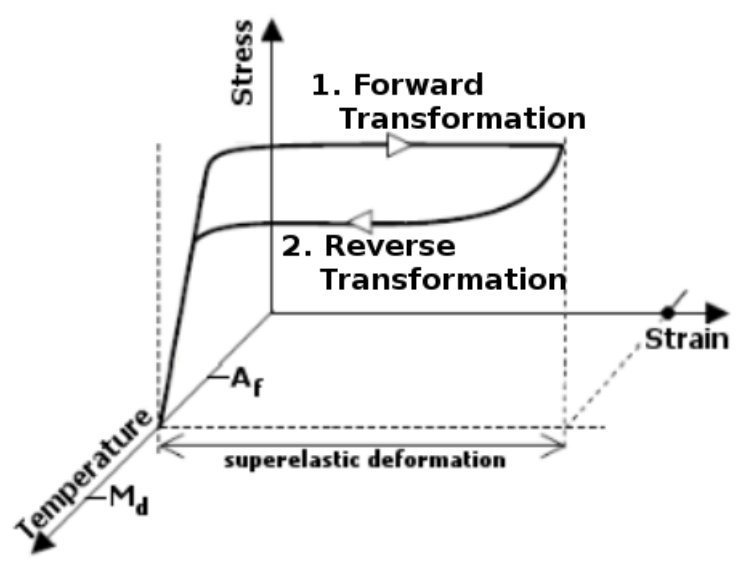

Fig. 2. Stress-strain characteristics exhibiting the superelasticity effect [8].

To understand the origin of high significance of the SMA properties, one should focus on the changes of characteristics shown in Fig. 2. The most crucial is the region where the stress-strain characteristics appoints constant stress field under wide strain field changes during phase transformations (a plateau region). Therefore, a SMA device can generate constant force while the displacement varies in the assumed range. Thanks to this phenomenon, SMA was successfully implemented in commercial applications such as medical staples and dental braces [10].

The unique behaviour of SMA is very attractive to be employed in unconventional cases where rigorous demands related to the number of movable parts and occupied space are required. Therefore the SMA devices are successfully developed in both aerospace and spacecraft applications [11]. However, SMA as smart materials are still at the research stage of their development. There is a group of existing phenomena with incomprehensible physical explanations. Presently, there is a great deal of effort to implement an efficient SMA model which would be able to accurately consider the numerous physical observations. The number of existing models shows that there is a lot of work to reach the aim. More information about different approaches to numerical modelling of SMA can be found in [12].

The present paper consists of 5 sections. After introductory Section 1, Section 2 provides description of the experiments carried out to obtain material properties for the tested SMA wire. Section 3 addresses a numerical model developed by the authors used to study influence of boundary conditions, followed by the results of numerical analyses presented in Section 4. Final Section 5 summarizes the paper and draws the concluding remarks.

\section{Experiment: material properties determination}

Before the details regarding carried out experiments are shown, the authors wish to introduce the background for SMA model validation issues, highlighting their specificity. Practical aspects of SMA modelling in the context of experimental activities are addressed in the following.

Most of commercial Finite Element Method (FEM) software packages have implemented SMA models. These models are often able to simulate only the phenomenon of superelasticity - as it is accessible in the ANSYS [13]. On the other hand, the MSC.Software MARC library contains two models of SMA, where the one-way memory effect is also implemented. However, one of them is the Aurrichio's model from 2001 [14], while the second one is the Saeedvafa's and Asaro's model proposed in 1995 [15]. It should be, however, noted that, a lot of new models have been proposed over more than 15 years, some of them employ more complex framework. Nevertheless, none of them was implemented in the mentioned FEM software packages. This can be seen as a determinant that reflects the difficulty of implementing such complex material models. Moreover, one should note that among all widely used approaches related to the SMA modelling [16], the FEM programs have implemented only the phenomenological models.

\subsection{Experimental uncertainty for material parameters identification}

One of the disadvantages of the phenomenological models is necessity to define numerous input parameters. In general, these parameters are divided into 3 groups:

- elastic parameters of both martensite and austenite such as: Young's modulus, Poisson's number, thermal expansion coefficient etc.,

- functions governing phase transformation caused by mechanical constraints, 
- functions governing phase transformation caused by thermal constraints.

The most popular SMA material is Nitinol, which is a nickel and titanium based alloy. Another example is Flexinol, which has the same chemical composition as Nitinol, but different manufacturing process causes that smaller wire diameters are possible to be achieved. In both cases the literature review provides a wide range of the elastic parameters values, e.g. the Young's modulus for austenite is found to be in the range of $60-83 \mathrm{GPa}$ for Nitinol [17]. The reason for this fact may be related to various alloy additions - used in order to change some material properties such as values of the characteristic temperatures. The side effect of the alloy composition variation can be a significant change of the elastic properties. From the other side, the above mentioned observations may lead to necessary adaptation and use of different experimental procedures. Lack of datasheets on the SMA properties as well as lack of appropriate norms and standards are the factors which aggravate the problem of the material parameters uncertainty. In case of tensile tests, one should note the existence of the ASTM norm [18], which was created mainly due to medical restriction reasons. This 6-page document contains only the description of methods used to determine the material parameters for the superelasticity at room temperature. Moreover, most of researches use standard grips during the tensile tests. These components hold specimens in position by generation the compression force. The aim of this paper is to prove that this kind of constraints could have negative impact on reliability of experimental results.

\subsection{Experiment: superelasticity in Flexinol wire}

Considering the above statements, a uniaxial tensile test was performed for a Flexinol wire with diameter of $0.51 \mathrm{~mm}$ and length of $50 \mathrm{~mm}$ for which the temperature $\mathrm{A}_{\mathrm{f}}$ equals $90^{\circ} \mathrm{C}$. The experimental test stand contains a fatigue testing machine Instron8872 with maximal load $10 \mathrm{kN}$ and a heating chamber Instron 3119-505 with temperature control in the range $-70^{\circ} \mathrm{C}$ to $300^{\circ} \mathrm{C}$. However, the most important, from the research point of view, is the information about used grips. The scheme and schematically presented grip operation are shown in Fig. 3 and 4, respectively.

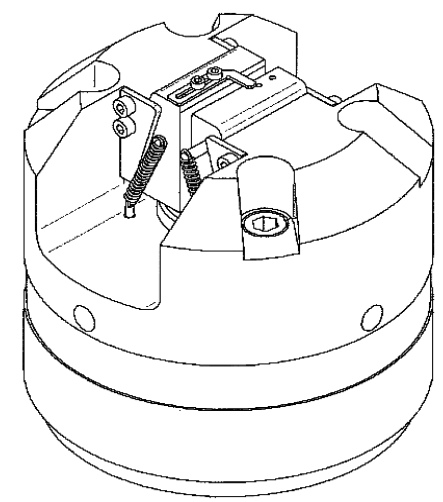

Fig. 3. Scheme of the grips [19].

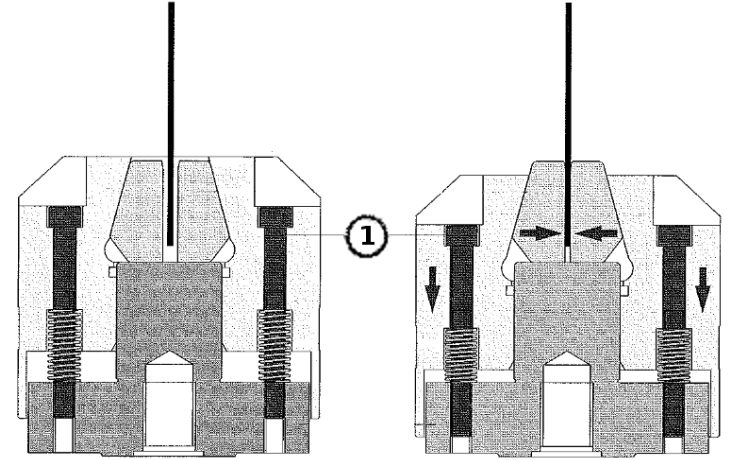

Fig. 4. The principle of the grip operation [19].

As shown in Fig. 4, tightening the screws 1 leads to closing the jaws. This method allows using a single grip for wide range of the specimen thicknesses. Moreover, the jaws are exchangeable and the various variants of their contact surfaces may be provided, which gives the opportunity to fit the experimental condition to demanded requirements. Taking into account the small diameter of the used Flexinol wire the authors used the most convenient grip configuration, which uses the jaws with serrated contact surfaces, as shown in Fig. 5.

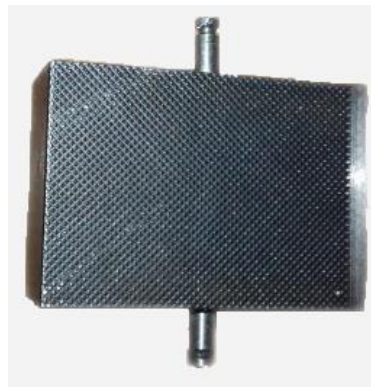

Fig. 5. Serrated jaws used to hold Flexinol wire

The intention of the authors was to perform the test following the most common condition applied by the research community. The similar system's configuration was used in many top works as [8], even for the high strain rates tests [20].

For the prepared measurement system, the test procedure was next defined. The used configuration gives an opportunity to control both the displacement of the grips as well as the force exerted on a tested specimen. The value change paths with respect to time obtained for the two mentioned parameters for three tests performed at $100^{\circ} \mathrm{C}$ are shown in Fig. 6. The displacement path is recorded as relative value where 0 is assigned to the initial position of the top grip. According both characteristics presented in Fig. 6, one can realize that the experiment contains six intervals:

1. pause for 5 seconds;

2. preloading sample with force of $10 \mathrm{~N}$ in order to cancel the slacks - load rate: $10 \mathrm{~N} / 30 \mathrm{sec}$;

3. pause for 5 seconds;

4. elongation of the sample up to $2.5 \mathrm{~mm}$ - load rate: $0.05 \mathrm{~mm} / \mathrm{s}$

5. pause for 5 seconds;

6. return of the top grip to the position from the step 3 - the same load rate applies as in the step 4. 

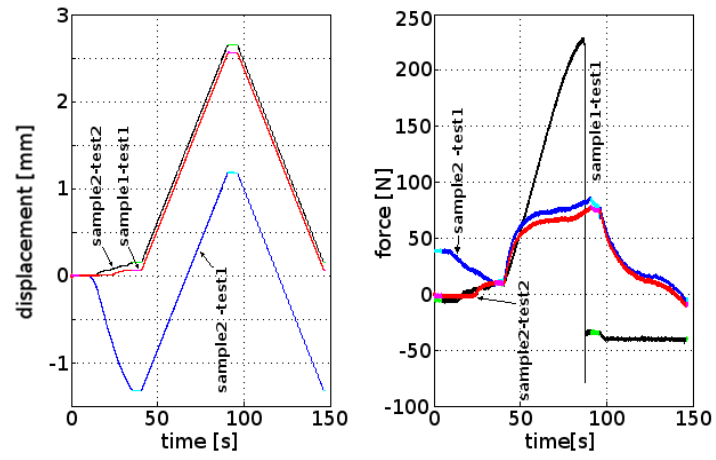

Fig. 6. The displacement of the grips (left) and the force exerted on a tested specimen (right).

As one can observe, the essential part of the test is the control process utilized by the displacement path the mentioned steps 4-6. However, the initial position of the sample is set by the $10 \mathrm{~N}$ force first. Therefore, the displacement path with respect to time is presented again according to the proper absolute initial position (reached at the end of step 2). The characteristics are shown in Fig. 7. Now, it is clear that for each experiment the top grip reaches the assumed displacement values from the steps 4 and 6 .

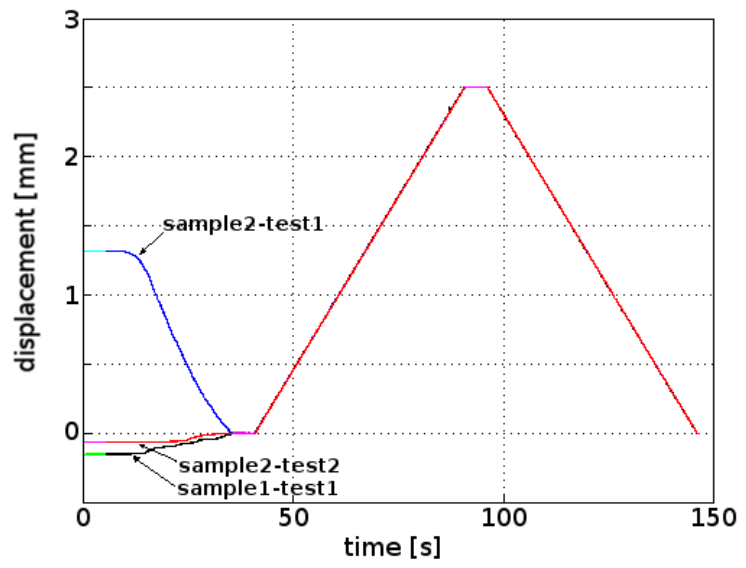

Fig. 7. The displacement path with respect to time according to the absolute initial position.

In the force-time characteristics in Fig. 6, it can be observed that the first sample was damaged during the test. This is one of the reasons why the essential parts of the tests were achieved by displacement controlling due to the safety reasons. There should be also noted that the first sample was used earlier during development of the test procedure. It means that the Flexinol wire was subjected to cyclic loading (as in case of fatigue), during which the local stress field propagates within the whole body of the specimen. Moreover, the cut off part of the sample does not lose the shape memory features like the one-way shape memory effect. According to the above presented statement, the most likely reason of the specimen damage was the influence of the sharp edge of the jaw, which is located between serrated and a flat surface of the contact area - see Fig. 5.

The results for the second sample are analysed according to the force-displacement characteristics shown in Fig. 8. The hysteresis does not close because of two reasons: too high initial force as well as generation of compression stress at the end of the test sequence. Moreover, the hysteresis paths are slightly different for the two following tests. These two, recently mentioned phenomena interfere and lead to the two-way shape memory effects manifestation [8]. Summarizing, reduction of the initial force and usage of the force control instead of the displacement control during the step 6 should be sufficient to improve the observed results.

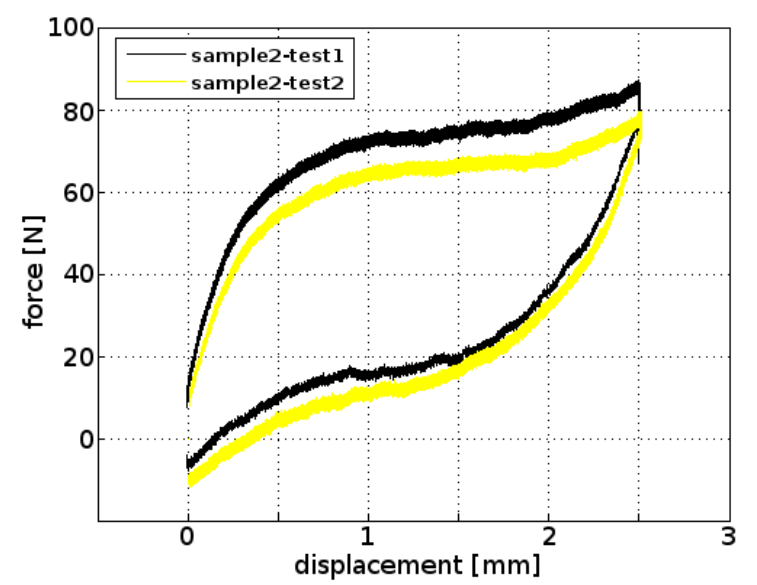

Fig. 8. The force-displacement characteristics of the experiments performed on the second specimen.

In the authors' opinion, the most important fact confirmed in the work, is that the presented results correctly project the superelasticity effect. Fig. 9 presents the stress-strain characteristics for the two considered tests. The values of the Young's modulus were calculated from the plots and they are $30.5 \mathrm{GPa}$ and $43 \mathrm{GPa}$ respectively for martensite $\left(\mathrm{E}^{\mathrm{M}}\right)$ and austenite $\left(E^{A}\right)$. The values fall within the range given in the literature, thereby confirming the correctness of the results.

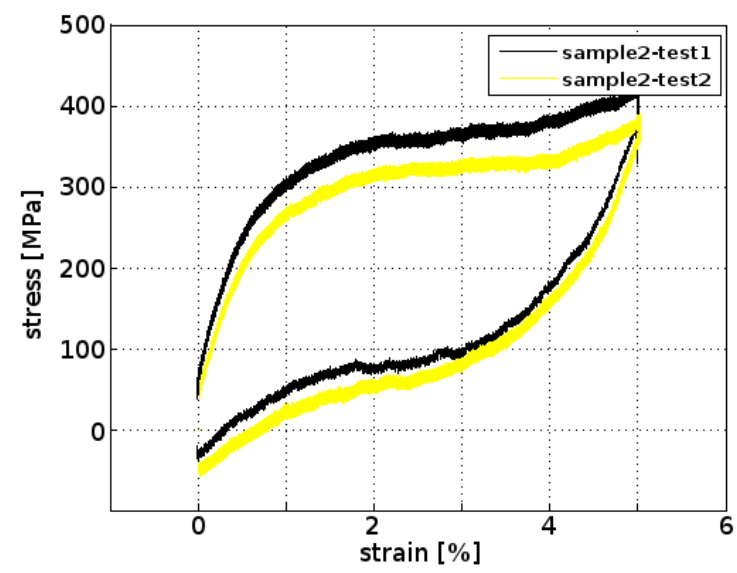

Fig. 9. The stress-strain characteristics of the experiments performed on the second specimen.

The second sample was subjected to 12 test cycles and all of the material responses convergence. On the other hand, the first sample was damaged after third test cycle. Both specimens were clamped in the same manner and with the same range of force and displacement 
values. The compression force generated by the grips is the only parameter out of the control, which can affect the test conditions considerably. As stated above, the hold strength is controlled by tightening the screw shown in Fig. 4. The disadvantage of this method is lack of a feedback on the reached value of the compression force. Thereby, the impact of this parameter on the material response is analysed in the simulation stage, presented in next sections.

\section{Model of the tested system: SMA sample and grips of fatigue testing machine}

The goal of the work is to reflect the experimental test conditions in the simulation software - MSC.Software MARC. Various parts of the measurement system were taken into account. There should be noted that presented SMA analysis considers strong nonlinearity because of specificity of the material behaviour as well as contact conditions. As a matter of fact, the number of FEs should be controlled to reach both result's convergence and acceptable computational time. Therefore, only half of the system is modelled based on symmetry of the model. Moreover, only the jaws and specimens are modelled as deformable bodies. The rest of the machine parts are assumed as rigid bodies due to their large masses and volumes compared to the parameters of the tested Flexinol wire. The final model (shown in Fig. 10) was created using the interface MSC.Software MENTANT. The way how the jaws were built is also presented in the plot to show the effort of minimisation the number of FEs. Finally, the model contains over 24500 FEs, where 17000 FEs represent the Flexinol wire.

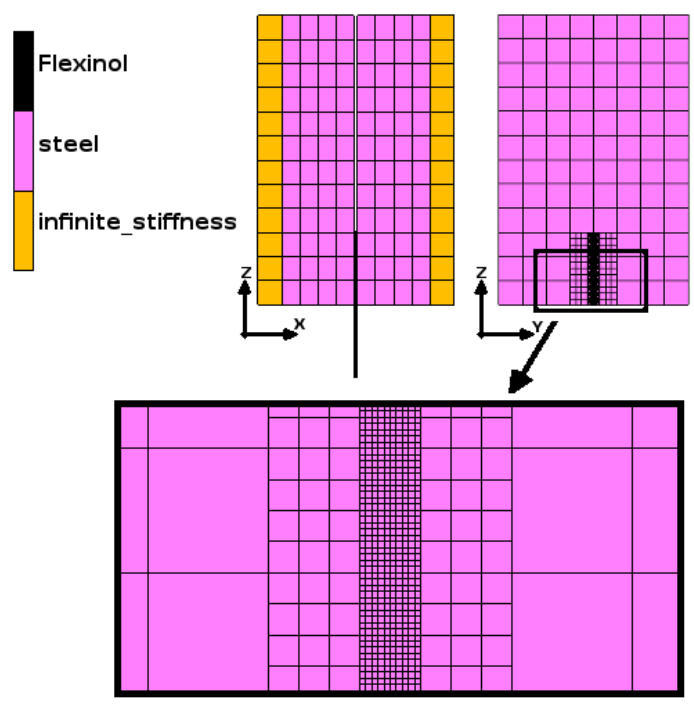

Fig. 10. Model of the tested system.

Simulation of the experimental work requires definition of two load cases: first one to analyse the jaws compression and second one to reflect the sample elongation. Therefore, proper boundary conditions must be defined. The functions used to describe the two forces, responsible for wire compression by the jaws and its further elongation, are crucial when obtaining required outcomes - which would confirm a hysteretic character of the material response. There should be mentioned that quasistatic analyses were performed. The constraint paths referring to the simulation steps for both above mentioned boundary conditions are shown in Fig. 11. Compression of the jaws was controlled with the displacement constraint, following the principle of grip operation - as seen in Fig. 4. The maximum value of this parameter, i.e. the displacement while compression of the jaws, is changed at each simulation, while the force path remains unchangeable. One can observe that the first load case lasts for 10 steps, while the second covers 400 steps. The reason of this situation is imposed by the convergence requirement of the analysis.

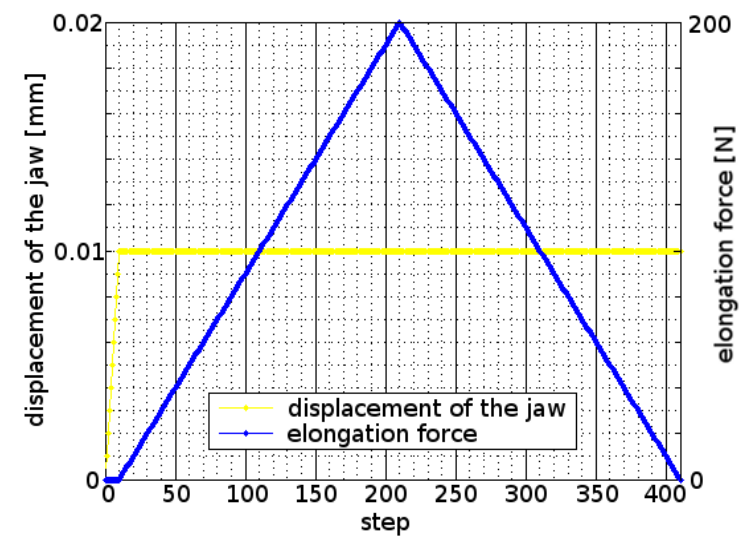

Fig. 11. The simulation constraints: displacement of the jaw and elongation force.

The simulation part of the paper is a theoretical one a validation process was not performed yet. Therefore, the material parameters and input model data took the recommended values - after [17]. Only the Young's modulus values were changed according to the validated values, as described in Section 2.2, to improve the results. By default, these parameters are equal in the MARC software. Table 1 presents the most important input data used in the model.

Table 1. Input parameters

\begin{tabular}{|c|c|}
\hline Parameter & Value \\
\hline $\begin{array}{c}\mathrm{E}^{\mathrm{A}} \\
\text { Young's modulus for austenite }\end{array}$ & $43.297 \mathrm{GPa}$ \\
\hline $\begin{array}{c}\mathrm{E}^{\mathrm{M}} \\
\text { Young's modulus for martensite }\end{array}$ & $30.455 \mathrm{GPa}$ \\
\hline $\begin{array}{c}v \\
\text { Poisson's number }\end{array}$ & 0.3 \\
\hline$\sigma_{\mathrm{s}}{ }^{\mathrm{AS}}$ & $500 \mathrm{MPa}$ \\
\hline$\sigma_{\mathrm{f}}^{\mathrm{AS}}$ & 500 \\
\hline$\sigma_{\mathrm{s}}{ }^{\mathrm{SA}}$ & 300 \\
\hline$\sigma_{\mathrm{f}}^{\mathrm{SA}}$ & 300 \\
\hline$\varepsilon$ \\
\hline Transformation Strain
\end{tabular}


The four stress parameters present in Table 1 mean respectively:

- $\sigma_{\mathrm{s}}^{\mathrm{AS}}$ - tensile stress for which transformation from austenite to martensite begins;

- $\sigma_{\mathrm{f}}^{\mathrm{AS}}$ - tensile stress for which transformation from austenite to martensite ends.

- $\sigma_{\mathrm{s}}^{\mathrm{SA}}$ - tensile stress for which transformation from martensite to austenite begins;

- $\sigma_{\mathrm{f}}^{\mathrm{SA}}$ - tensile stress for which transformation from martensite to austenite finishes.

\section{Numerical results}

In this section the superelasticity effect is numerically simulated in tensile tests. The studies are divided into two parts: first one presents the results for a theoretical elongation, while second one is devoted to the experiments in fatigue testing machine.

\subsection{Theoretical results}

The theoretical elongation case was studied by including only the SMA wire in the simulation. Therefore, the first load case described in Section 3 was omitted and the specimen was only strengthened by forces applied to its ends. The simulation was performed for a $10 \mathrm{~mm}$-long SMA wire. Finally, the force and displacement data was obtained from the result file for each step. The identified characteristics are shown in Fig. 12. The obtained results are considered as reference for further analysis. Thereinafter, the mentioned relation will be recalculated to presents stress-strain characteristics due to their more general form, which better reflects the material behaviour.

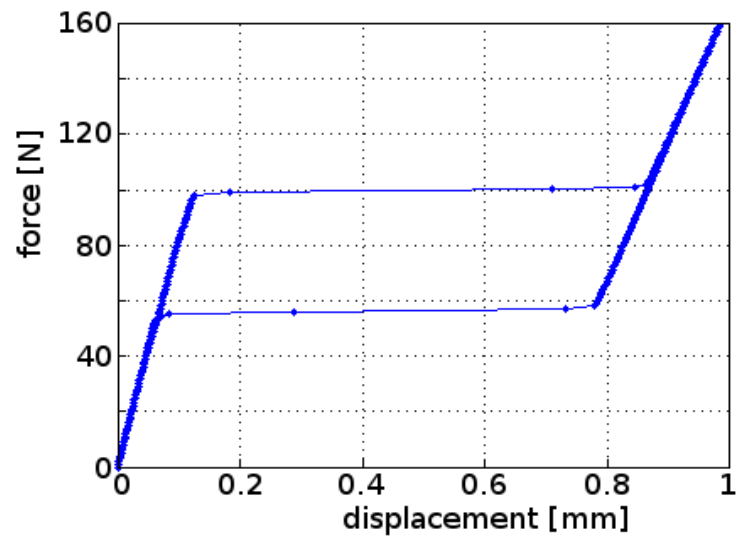

Fig. 12. The force-displacement characteristics of the theoretical elongation - simulation results.

\subsection{Simulation of elongation experiments with use of fatigue testing machine}

Five simulations were performed for different maximum values of the compression force applied during the first load step. As mentioned in Section 3 the boundary condition's constraint is defined by the displacement path. The maximum values for the performed analyses are in the range $[0.01,0.05 \mathrm{~mm}$ ] with the increment step
$0.01 \mathrm{~mm}$. The force path defined for the second load step remains the same for all cases and is shown in Fig. 11.

The obtained results are compared with the referential ones, for which a theoretical case was solved. The simulations were performed for a $200 \mathrm{~mm}$-long SMA wire, which is clamped between jaws along the longitudinal distance of $10 \mathrm{~mm}$. Therefore, in all simulations the $10 \mathrm{~mm}$-long part of the specimen is elongated which gives opportunity to perform a quantitative analysis. Fig. 13 presents the stress-strain characteristics from all simulations.

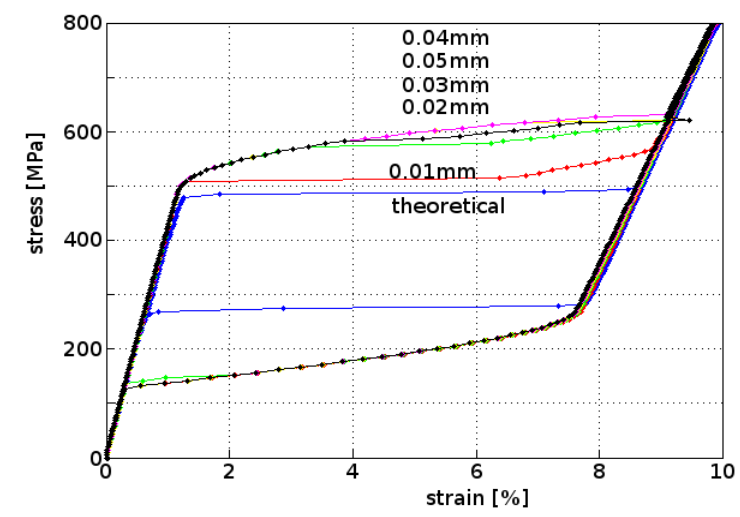

Fig. 13. The stress-strain characteristics for all simulations node located at the specimen central longitudinal axis.

Starting from the initial point of the curves seen in Fig. 13, i.e. at the coordinates $(0,0)$, we can observe that the Young's modulus for elastic strain is a little higher than the ones found for the theoretical case and the value of $\sigma_{\mathrm{s}}{ }^{\mathrm{AS}}$. The last four simulations show a nonlinear behaviour for the transformation path and much higher stress value where it finishes. The exception is the first case where the maximum displacement of the jaws is $0.1 \mathrm{~mm}$. In this simulation the transformation path is similar to the theoretical one for major part of the path. Therefore, the behaviour of the specimen is changed during the transformation process. The stress-strain characteristics freezes at a specific level of the stress value equal $519 \mathrm{MPa}$ to be almost parallel to the strain axis, what takes place in the 115-th step of the simulation. From this purpose, Fig. 14 presents the contribution percentage of martensite in the material for steps from 114 to 122 . Fig. 14 contains information about values of the parameters for the SMA wire close to the contact area. The results are presented for two planes: $\mathrm{XZ}$ and $\mathrm{YZ}$ - one of the grips was hidden to plot FEs of SMA wire at the second plane.

As one can observe, at the 115-th step the phase transformation is started in the whole body of specimen. This phenomenon appears on the stress-strain path by changing the slope of the curve. However, the phase transformation actually starts earlier. Fig. 14 presents the distribution of the martensite in material which is heterogeneous. The phase transformation begins at the contact area of the SMA wire and grips. The shown force-displacement as well as stress-strain characteristics present the results for node located on the longitudinal axis of the wire, i.e. for the coordinates $(0,0,10 \mathrm{~mm})$. Next, the analysis extends to the points placed at the wire circumference, i.e. $(0,0.255,10 \mathrm{~mm})$ as well as 
$(0.255,0,10 \mathrm{~mm})$. The stress-strain characteristics for both nodes are respectively presented in Fig. 15 and 16, for all simulations.

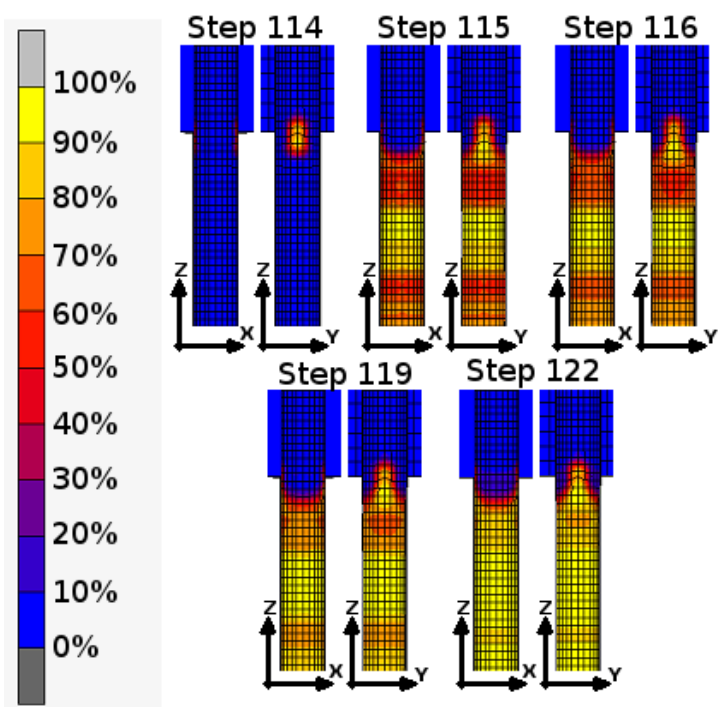

Fig. 14. The simulation results for first case $(0.01 \mathrm{~mm})-$ MENTAT visualisation of results presented in Fig. 13.

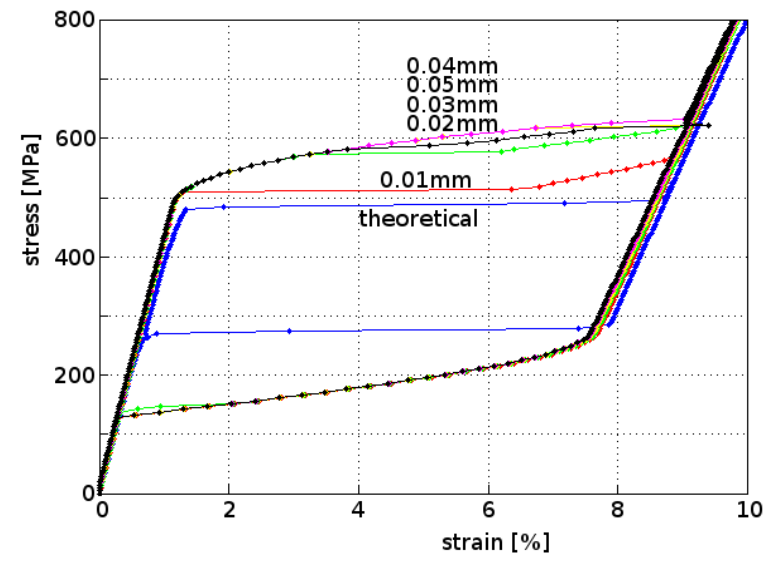

Fig. 15. The stress-strain characteristics for all simulations node located at the wire circumference and $\mathrm{X}$ axis.

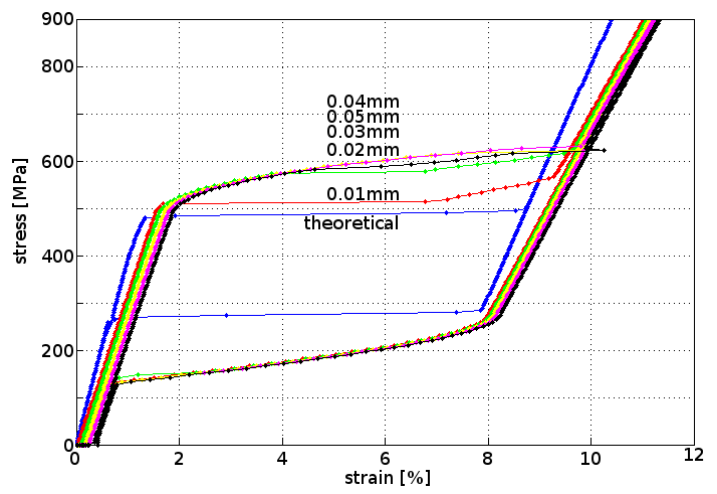

Fig. 16. The stress-strain characteristics for all simulations node located at the wire circumference and $\mathrm{Y}$ axis.

By comparison of Fig. 13 and 15-16 one can see that the first two mentioned characteristics are almost the same, while the last one is significantly different from the previous ones. In the last shown relationship (Fig. 16) the initial strains are observed due to compression force generated by the jaws. Moreover, different stiffness value is observed for elastic strain at the area of the highest stress value (as shown in Fig. 14).

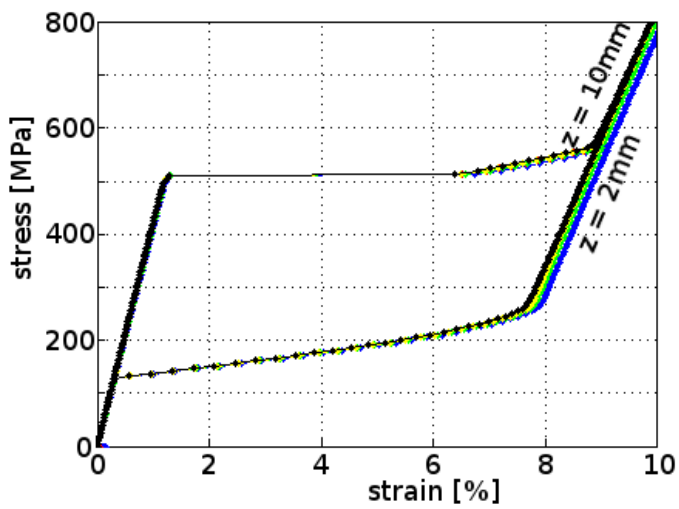

Fig. 17. The stress-strain characteristics for first simulation $(0.01 \mathrm{~mm}$ displacement of jaws $)$ - nodes located at the specimen $Z$ coordinates: $\{2,4,6,8,10 \mathrm{~mm}\}$.

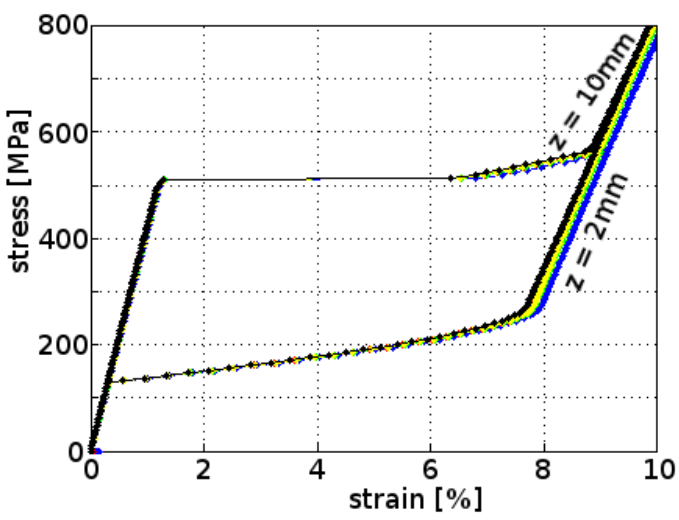

Fig. 18. The stress-strain characteristics for first simulation $(0.01 \mathrm{~mm}$ displacement of jaws $)$ - nodes located at the wire circumference and $\mathrm{X}$ axis with $\mathrm{Z}$ coordinates: $\{2,4,6$, $8,10 \mathrm{~mm}\}$.

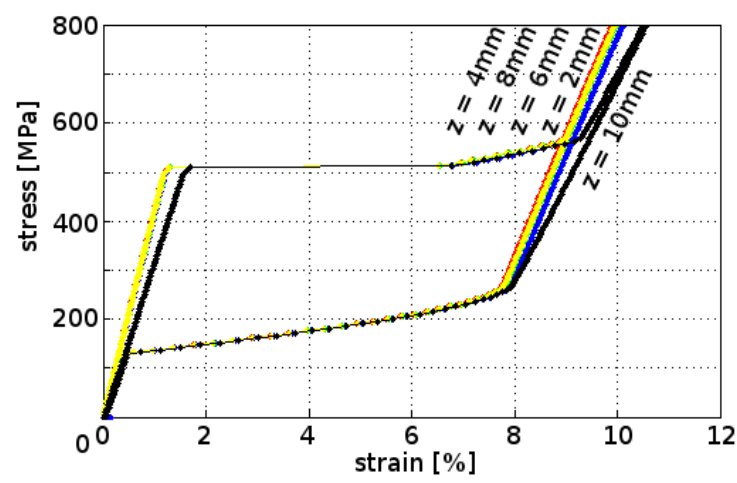

Fig. 19. The stress-strain characteristics for first simulation $(0.01 \mathrm{~mm})$ - nodes located at the wire circumference and $Y$ axis with $Z$ coordinates: $\{2,4,6,8,10 \mathrm{~mm}\}$.

Fig. 14 confirms that the martensite distribution is heterogeneous not only for the points placed on the wire circumference, but also along $Z$ axis. Therefore the results for other nodes placed in the whole body of SMA wire are also presented. Exemplary characteristics are shown only for the simulation with $0.01 \mathrm{~mm}$ displacement of the jaws. Hence: 
- $\quad$ Fig. 17 presents the results for nodes with coordinates $(0,0)$ on the XY plane;

- Fig. 18 presents the results for nodes with coordinates $(0,0.225 \mathrm{~mm})$ on the XY plane;

- $\quad$ Fig. 19 presents the results for nodes with coordinates $(0.225 \mathrm{~mm}, 0)$ on the XY plane;

Each figure contains the characteristics for nodes with the coordinate along $\mathrm{Z}$ axis from the set: $\{2,4,6,8$, $10 \mathrm{~mm}\}$.

\section{Summary and concluding remarks}

The purpose of the study is to verify the influence of boundary conditions on the superelasticity effects occurring in SMA. Hence, the tensile test procedure was modelled in FEM software. The simulation includes contact conditions between the jaws of the grips and wire made of SMA as well as implementation of boundary conditions reflecting real constraints which appear during experimental tests. The theoretical elongation was simulated to obtain the referential data for the other models. Finally, five models were defined with different values of compression force generated by the grips. Therefore, six simulation cases were studied.

As expected, the compression force affects the local phase transformation in the SMA specimen significantly. Initially, the authors expected that martensite fraction occurrence at the beginning of the simulation would lead to decrease of the wire stiffness value. However, the Young's modulus for elastic region of the stress-strain curve is slightly higher than the one for austenite for all performed simulations. On other hand, in the martensite occurrence region the elastic modulus is lower than for the mentioned phase. The authors consider the influence of geometry of the deformed wire on the stiffness factor as one of possible explanation for the above mentioned observation. However, the analysis should be continued to verify the proposed origin of the phenomenon. Moreover, other aspects in superelasticity behaviour which require explanation are indicated:

- significant increase of dissipation energy opportunity which is proportional to the size of the area enclosed by hysteresis (seen in Fig. 15 and 16);

- changes of the values of $\sigma_{\mathrm{f}}^{\mathrm{AS}}$ as well as $\sigma_{\mathrm{f}}^{\mathrm{SA}}$, while two others $\left(\sigma_{\mathrm{s}}{ }^{\mathrm{AS}}\right.$ and $\left.\sigma_{\mathrm{s}}{ }^{\mathrm{SA}}\right)$ remain almost constant;

- nonlinear shape of transformation curve (a plateau region for the stresses, e.g. seen in Fig. 15 and 16).

There should be noted that the results obtained for the case with prestress (due to jaws compression) are more similar to the experimental response, presented in Section 2, than the theoretical one. That should be considered as motivation to continue the study. However, future validation is essential to gain quantitative dependencies which could confirm the above stated ideas.

This work has been supported by the AGH University of Science and Technology, WIMiR, research grant no. 15 .11 .130 .633 and 11.11 .130 .560 .

\section{References}

1. M. Jianzuo, H. Haolei, H. Jin, Adv. in Mat. Sc. And Eng., 1-6 (2013)

2. F. Auricchio, E. Bonetti, G. Scalet, F. Ubertini, Int. J. Plast., 59 (2014)

3. D. Lagoudas, P. Entchev, P. Popov, E. Patoor, L. Brinson, X. Gao, Mech. Mater., 38 (2006)

4. J. Bryła, A. Martowicz, Projektowanie mechatroniczne: zagadnienia wybrane, (AGH, 2016)

5. A. Martowicz, J. Bryła, T. Uhl, Uncertainty quantification for the properties of a structure made of SMA utilising numerical model (ISMA2016 \& USD2016)

6. J. Bryła, P. Palenica, A. Martowicz, Projektowanie mechatroniczne: zagadnienia wybrane (AGH, 2016)

7. J. Bryła, A. Martowicz, Mechanics\&Control, 34:3, (2015) (in press)

8. D. Lagoudas, Shape Memory Alloys: Modeling and Engineering Applications (Springer, 2008)

9. F. Aurcchio, D. Fugazza, R. DesRoches, J. Eng. Mat. and Tech., 128 (2006)

10. L. Lecce, A. Concilio, Shape Memory Alloy Engineering, Butterworth Heinemann, Chapter 11, (2014)

11. O. Godard, M. Lagoudas, D. Lagoudas, Smart Struct. and Mat., 5056 (2003)

12. C. Cisse, W. Zaki, T. Zineb, Smart Mater. and Struct., 10, (2016)

13. S. Imaoka, ANSYS Tech. Supp. Gr., 4 (2014);

14. F. Auricchio, Int. J. Pl., 17 (2001);

15. M. Saeedvafa, R. Asaro, Los Alamos Report (1995)

16. A. Khandelwal, V. Buravalla, Int. J. Struct., 1,111148 (2009)

17. Marc Volume A: Theory and User Information. Release Marc2015 (2015);

18. ASTM International, Standard Test Method for Tension Testing of Nickiel-Titanium Superelastic Materials (2006)

19. Fatigue Testing Machine documentation (2017)

20. A. Vitiello, G. Giorleo, R. Morace, Smart Mater. Struct., 14 (2015). 